\title{
Inbreeding among Caribbean Hispanics from the Dominican Republic and its effects on risk of Alzheimer disease
}

\author{
Badri N. Vardarajan, PhD ${ }^{1,2}$, Daniel J. Schaid, PhD ${ }^{3}$, Christiane Reitz, MD, PhD ${ }^{1,2,4,5}$, \\ Rafael Lantigua, MD ${ }^{1,6}$, Martin Medrano, MD', Ivonne Z. Jiménez-Velázquez, MD, \\ Joseph H. Lee, PhD ${ }^{1,2,4}$, Mahdi Ghani, PhD ${ }^{9,10}$, Ekaterina Rogaeva, PhD ${ }^{9,10}$, \\ Peter St George-Hyslop, MD ${ }^{9-11}$ and Richard P. Mayeux, MD $1,2,4,5,12$
}

\begin{abstract}
Background: Inbreeding can be associated with a modification of disease risk due to excess homozygosity of recessive alleles affecting a wide range of phenotypes. We estimated the inbreeding coefficient in Caribbean Hispanics and examined its effects on risk of late-onset Alzheimer disease.
\end{abstract}

Methods: The inbreeding coefficient was calculated in 3,392 subjects (1,451 late-onset Alzheimer disease patients and 1,941 age-matched healthy controls) of Caribbean Hispanic ancestry using 177,997 nearly independent single-nucleotide polymorphisms from genomewide array. The inbreeding coefficient was estimated using the excess homozygosity method with and without adjusting for admixture.

Results: The average inbreeding coefficient in Caribbean Hispanics without accounting for admixture was $F=0.018( \pm 0.048)$, suggesting a mating equivalent to that of second cousins or second cousins once removed. Adjusting for admixture from three parent populations, the average inbreeding coefficient was found to be $0.0034( \pm 0.019)$ or close to third-cousin mating. Inbreeding coefficient was a significant predictor of Alzheimer disease when age, sex, and APOE genotype were used as adjusting covariates $(P=0.03)$.

Conclusion: The average inbreeding coefficient of this population is significantly higher than that of the general Caucasian populations in North America. The high rate of inbreeding resulting in increased frequency of recessive variants is advantageous for the identification of rare variants associated with late-onset Alzheimer disease.

Genet Med advance online publication 13 November 2014

\section{INTRODUCTION}

Inbreeding can lead to rare heritable illnesses conferred by homozygous recessive alleles. Reduced early survival of children from first-cousin marriages and similar observations in other organisms emphasize the presence of an increased number of homozygous deleterious alleles in the genome. ${ }^{1,2}$ Inbreeding is highly prevalent across the world, and differences in disease prevalence between populations can be partially attributed to extent of inbreeding. ${ }^{1,3}$

Conservative estimates of prevalence of consanguineous marriages (defined as a union between individuals related as second cousins or closer) range between 1 and 10\% among 2,811 million people studied globally. ${ }^{3}$ Previous studies have seen a strong association between the extent of inbreeding and reproductive health, as well as childhood mortality and rare Mendelian disorders. ${ }^{1,2,4,5}$ However a multipopulation metaanalysis found only a moderate $1.1 \%$ increase in the infant death rate of $1.1 \%$ in the progeny of first cousins. ${ }^{6}$ Biological consequences of inbreeding are known to become worse with aging in nonhuman species. ${ }^{7,8}$ Studies examining the effects of inbreeding on late-onset complex diseases have found conflicting trends. Longer stretches of homozygosity have been observed in patients with breast, prostate, head and neck, and colorectal cancer, ${ }^{9,10}$ but these findings have not been consistently replicated. ${ }^{11-14} \mathrm{~A}$ recent study examining the concordance of Alzheimer disease (AD) raised the possibility that as much as $90 \%$ of early-onset cases with $\mathrm{AD}$ are probably the result of autosomal recessive inheritance. ${ }^{15}$ Multiple risk loci for $\mathrm{AD}^{16}$ were detected in a consanguineous Israeli-Arab community from WadiAra (Israel). Unaffected healthy controls were found to be more inbred than cases in the WadiAra population, suggesting higher frequency of protective alleles as a result of inbreeding. ${ }^{17}$ In an autopsy-confirmed AD data set comprising subjects from the Saguenay region of Quebec (Canada), subjects with lateonset $\mathrm{AD}$ and having at least one $A P O E \varepsilon 4$ allele were observed to have higher levels of inbreeding (equivalent to first-cousin

\footnotetext{
${ }^{1}$ The Taub Institute for Research on Alzheimer's Disease and the Aging Brain, Columbia University, New York, New York, USA; ${ }^{2}$ The Gertrude H. Sergievsky Center, Columbia University, New York, New York, USA; ${ }^{3}$ Mayo Clinic, Rochester, Minnesota, USA; ${ }^{4}$ Department of Epidemiology, Mailman School of Public Health, Columbia University, New York, New York, USA; ${ }^{5}$ Department of Neurology, Columbia University, New York, New York, USA; ${ }^{6}$ Department of Medicine, College of Physicians and Surgeons, Columbia University, New York, New York, USA; ${ }^{7}$ School of Medicine, Pontificia Universidad Catolica Madre y Maestra, Santiago, Dominican Republic; ${ }^{8}$ Geriatrics Program, Internal Medicine Department, School of Medicine, University of Puerto Rico, San Juan, Puerto Rico; ${ }^{9}$ Tanz Centre for Research in Neurodegenerative Diseases, University of Toronto, Toronto, Ontario, Canada; ${ }^{10}$ Department of Medicine, University of Toronto, Toronto, Ontario, Canada; ${ }^{11}$ Department of Clinical Neurosciences, Cambridge Institute for Medical Research, University of Cambridge, Cambridge, UK; ${ }^{12}$ Department of Psychiatry, School of Public Health, Columbia University, New York, New York, USA. Correspondence: Richard P. Mayeux (RPM2@columbia.edu)
} 
genomic sharing) compared with healthy controls. ${ }^{18}$ Recent studies have found the presence of long runs of homozygosity (ROHs) in Caribbean Hispanic late-onset Alzheimer disease (LOAD) patients as compared with healthy controls. ${ }^{19}$ By contrast, two ROH studies of outbred Caucasian populations did not yield any significant associations with LOAD ${ }^{20,21}$ In the current study, we estimated the level of inbreeding in Caribbean Hispanic families that are known to have higher rates of LOAD ${ }^{22}$ and investigated the association between inbreeding and risk of LOAD. Potential association between inbreeding and risk of LOAD may aid next-generation sequencing studies in mapping of disease-related genes.

\section{MATERIALS AND METHODS}

\section{Study population}

Study participants were identified from two source populations of Caribbean Hispanic ancestry. The two-parent studies include the Washington Heights-Inwood Columbia Aging Project (WHICAP) ${ }^{23}$ and the Estudio Familiar Influencia Genetica en Alzheimer (EFIGA) family study. ${ }^{24}$ The WHICAP study is a longitudinal cohort study that has examined a multiethnic cohort of elderly individuals residing in northern Manhattan (New York, New York). We recruited Medicare recipients who were at least 65 years of age, were without dementia, and lived in three contiguous postal codes in northern New York City. The EFIGA study is a family-based study comprising Dominican families with multiple persons affected with LOAD and a casecontrol study that included unrelated patients with LOAD and similarly aged unaffected and unrelated controls. Study participants were recruited from multiple sources including clinics in the Dominican Republic, as well as the Alzheimer's Disease Research Center Memory Disorders Clinic at Columbia University in New York City. To augment family recruitment, we advertised in local newspapers and media in the Dominican Republic and New York. In addition, we recruited probands from the WHICAP study when the informant reported family members with dementia. Families were recruited as follows: once probands were identified, structured family history interviews were conducted to determine whether siblings and more distant relatives were affected with dementia. When probands had $\mathrm{AD}$ and also had other family members with dementia, we interviewed and neurologically evaluated all siblings and more relatives. We assessed and corrected for cryptic relatedness by using genetic markers. The Caribbean Hispanic case-control study complements the EFIGA study in that the sampling frame was the same as that of the EFIGA study; however, recruitment was restricted to affected and unaffected persons who were unrelated and did not have family history of dementia. For these participants, we performed the same extensive medical, neurological, and neuropsychological evaluations at each visit. Clinical diagnoses were made in a consensus diagnostic conference by a panel of neurologists, neuropsychologists, and psychiatrists. The presence of LOAD was assessed based on the criteria established by the National Institute of Neurological and Communicative Disorders and Stroke and the Alzheimer's
Disease and Related Disorders Association. ${ }^{25}$ Additional demographic and epidemiological information was available for all genotyped individuals (Table 1). For estimating inbreeding in Caribbean Hispanics in this study, we selected age-matched unrelated cases and controls from the WHICAP and the EFIGA studies and one case per family from the EFIGA study.

\section{Genotyping}

Previously, a HumanOmni-650Y SNP chip was used for genome-wide association study (GWAS) of 1,094 Caribbean Hispanics. ${ }^{26}$ This study consisted primarily of samples from whole blood, with $0.16 \%$ of samples from saliva. Blood samples were extracted using the Qiagen method; saliva samples were extracted using the Oragene method. Samples were genotyped in batches corresponding to 96-well plates. Each plate contained one or two HapMap controls, as well as an average of two study sample duplicates. The DNA samples were genotyped at the Center for Inherited Disease Research using the Illumina HumanOmni1-Quad Quad v1 $0 \mathrm{H}$ array (http://www. illumina.org) and using the calling algorithms GenomeStudio version 2011.1, Genotyping Module 1.9.4, and GenTrain version 1.0 (Illumina, San Diego, CA). The genome build is 37/hg19. We combined the previously published GWAS data with the samples genotyped on the Illumina HumanOmni1-Quad chip (Illumina) to create a large data set for estimating the degree of inbreeding in the Caribbean Hispanic population.

\section{GWAS quality control}

We used the quality assurance/quality control protocol described by Laurie et al. ${ }^{27}$ to ensure consistency of the data. We excluded from the analyses samples missing $2 \%$ of the singlenucleotide polymorphisms (SNPs) from the GWAS panel and SNPs with a genotype missingness rate of $5 \%$ or SNPs with a minor allele frequency $<0.05$.

\section{Statistical analyses}

We pruned the genome-wide SNP data based on linkage disequilibrium to retain 177,997 tagging SNPs from GWAS data at pairwise $r^{2}<0.3$ using PLINK. ${ }^{28}$ We estimated the inbreeding coefficient in the sample set using the GCTA software. ${ }^{29}$ GCTA gives two estimates for the relationship between haplotypes within an individual: one based on the variance of additive genetic values (diagonal of the SNP-derived genetic relationship matrix) and the other based on SNP homozygosity (implemented in PLINK). ${ }^{28}$ Here we report the second measure of inbreeding (the two metrics on average gave similar results). In the context of inferring relatedness in GWAS with population

Table 1 Demographic information

\begin{tabular}{lc} 
Characteristic & $\begin{array}{c}\text { Caribbean Hispanic } \\
\text { population }(\boldsymbol{n}=\mathbf{3 , 3 9 2})\end{array}$ \\
\hline Age, years, mean (SD) & $75.75(9.41)$ \\
Education, years, mean (SD) & $6.4(4.99)$ \\
Women, \% & 68 \\
Cases (\%) & $1,532(45.4)$ \\
\hline
\end{tabular}


Table 2 Inbreeding coefficient by ancestry of the samples

\begin{tabular}{lcccccc} 
& N & Unaffected & Affected & $\begin{array}{c}\text { Mean inbreeding } \\
\text { coefficient in all } \\
\text { samples }\end{array}$ & $\begin{array}{c}\text { Mean inbreeding } \\
\text { coefficient in } \\
\text { unaffected subjects }\end{array} \quad \begin{array}{c}\text { Mean inbreeding } \\
\text { coefficient in } \\
\text { affected subjects }\end{array}$ \\
\hline Asian & 41 & 30 & 11 & -0.0134 & -0.0146 & -0.0100 \\
Black & 356 & 153 & 202 & 0.0014 & 0.0018 & 0.0011 \\
Caucasian & 1,520 & 672 & 847 & 0.0066 & 0.0062 & 0.0069 \\
Mixed & 1,375 & 623 & 745 & 0.0005 & 0.0001 \\
\hline
\end{tabular}

Samples were classified as Asian, Caucasian, or African if they showed $>60 \%$ admixture from the parent population (the differences between unaffected subjects and affected subjects were not statistically significant in any of the three ethnic groups).

Table 3 Association of inbreeding with age

\begin{tabular}{|c|c|c|c|c|}
\hline Test & $\beta$ & SE & $z$ & $P$ \\
\hline $\begin{array}{l}\text { Age (in unaffected } \\
\text { subjects }>60 \text { years) } \sim F\end{array}$ & -24.17 & 12.86 & -1.88 & $6.05 \mathrm{E}-02$ \\
\hline Age at onset (in cases) $\sim F$ & -15.13 & 9.43 & -1.6 & 1.09E-01 \\
\hline $\begin{array}{l}\text { Age at onset } \sim F+\text { sex }+ \\
\text { APOE }(0,1,2)\end{array}$ & -12.28 & 9.24 & -1.33 & $1.84 \mathrm{E}-01$ \\
\hline
\end{tabular}

Effects of inbreeding on age in unaffected subjects and age at onset in affected LOAD cases were tested using a linear regression model.

$F$, inbreeding coefficient; LOAD, late-onset Alzheimer disease.

structure, relatedness estimation methods that assume population homogeneity can give extremely biased estimates. We used the Relatedness Estimation in Admixed Populations (REAP) software $^{30}$ to estimate the average inbreeding in the Caribbean Hispanic population by adjusting for their admixed ancestry. REAP takes as input the proportion of parental populations for each sample and estimates autosomal kinship coefficients and identity-by-descent sharing probabilities using SNP genotype data in samples with admixed ancestry. We estimated the proportion of ancestry from each parental population using the ADMIXTURE software ${ }^{31}$ by assuming that the admixture in the Caribbean Hispanics is conferred by two, three, four, and five parent populations. This software provided a maximum likelihood estimation of individual ancestries from multilocus SNP genotype data sets. We then used the estimates of parental population proportions and allele frequencies for each sample as input to the REAP software to compute admixture-adjusted inbreeding coefficients.

\section{RESULTS}

The average inbreeding coefficient in the Caribbean Hispanics without accounting for admixture (computed using GCTA software) was $0.018( \pm 0.048)$, suggesting significant inbreeding; $1,372(40.4 \%)$ of the 3,392 subjects had an inbreeding coefficient greater than 0.02 (Supplementary Figure S1 online). We computed the inbreeding coefficient accounting for admixture conferred to Caribbean Hispanics from two, three, four, and five parent populations (Supplementary Figure S2 online). Traditional methods assume homogeneity of population, which can significantly inflate inbreeding estimates. By using REAP, we adjust for subpopulation frequencies at sites to calculate admixture-adjusted inbreeding coefficients. It can be argued that Caribbean Hispanics are derived from Caucasian, African, Asian, and American Indian ancestries and the exact number of parental populations is unknown. Hence, we used three to five ancestral populations as input to adjust for admixture in Caribbean Hispanics (Supplementary Table S1 online). Adjusting for admixture, the average inbreeding coefficient decreased, ranging from $0.0034( \pm 0.019)$ for three parent populations to $0.002( \pm 0.018)$ for five parent populations. Of 3,392 samples, 329 (9.7\%) were highly inbred, with an inbreeding coefficient $F>0.02$, assuming admixture from three parent populations. Supplementary Figure $\mathbf{S} 3$ online shows the admixture in Caribbean Hispanics originating from Caucasian, African, and Asian ancestries. We used the admixture-adjusted inbreeding coefficient values for samples obtained from REAP software in subsequent analyses; $47.4 \%$ of the samples in the data set have predominantly Caucasian ancestry (Supplementary Figure $\mathbf{S 3}$ online). The mean inbreeding coefficient was highest among those of Caucasian ancestry $(F=0.0066$; equivalent to mating by second cousins once removed), followed by those with African ancestry $(F=0.0014$; Table 2). Supplementary Figure $\mathbf{S 4}$ online shows the overall distribution of the inbreeding coefficients in the samples by computed ancestry. Samples with African ancestry were less inbred than individuals having a significant proportion of the other two ancestries. Of those of Caucasian ancestry, $14 \%$ had a high inbreeding coefficient of $>0.02$ compared with $4.8 \%$ for individuals of Asian and $4.8 \%$ individuals of African ancestry.

We then tested the association of the inbreeding with age LOAD status and age at onset of the disease using logistic and linear regression models whenever suitable (Table 3). For each sample, we used the proportion of ancestry from the Asian and African parent populations (Caucasian ancestry was the reference value) as covariates. Age was weakly inversely correlated with the inbreeding coefficient but was not statistically significant.

Inbreeding was a significant predictor of LOAD adjusted for age, sex, and population covariates $(P=0.034$; Table 3$)$. The presence or absence of the APOE $\varepsilon 4$ allele when used as a covariate in the model strengthens the association of the inbreeding coefficient with $\operatorname{LOAD}(P=0.03)$. This could possibly imply that of the level of inbreeding is correlated with APOE $\varepsilon 4$ status and the residual effect of inbreeding level on LOAD risk after adjusting for $A P O E$ genotype could be attributable to other recessive loci. To test the relationship of $A P O E$ with inbreeding 
Table 4 Association of inbreeding with LOAD status

\begin{tabular}{lcccc} 
Test & $\boldsymbol{\beta}$ & $\boldsymbol{S E}$ & $\boldsymbol{Z}$ & $\boldsymbol{P}$ \\
\hline LOAD $\sim F$ & 3.88 & 2 & 1.95 & $5.17 \mathrm{E}-02$ \\
LOAD $\sim F+$ age + sex & 4.25 & 2.01 & 2.11 & $3.44 \mathrm{E}-02$ \\
LOAD $\sim F+$ age + sex + APOE $(0,1,2)$ & 4.39 & 2.02 & 2.17 & $3.02 \mathrm{E}-02$ \\
\hline
\end{tabular}

Effects of inbreeding on LOAD were tested using a logistic regression framework. All tests in Tables $\mathbf{3}$ and $\mathbf{4}$ are adjusted for covariates measuring the proportion of ancestral population (Caucasians, Asians, and Africans). The proportion of Caucasian ancestry was used as the base and two variables were used to specify the proportions of Asian ancestry and African ancestry.

$F$, inbreeding coefficient; LOAD, late-onset Alzheimer disease.

and its association with $\mathrm{AD}$, we regressed the inbreeding coefficient on $A P O E \& 4$ status in cases and controls separately. Inbreeding is associated with number of $\varepsilon 4$ alleles in unaffected subjects (Supplementary Table S2 online) but not in affected subjects. Also, the direction of effect was in the opposite direction in cases versus controls. Alternatively, extent of inbreeding tended to increase the $\varepsilon 4$ copies in cases but decreased $\varepsilon 4$ copies in controls. This prompted us to test an interaction model between $A P O E$ and inbreeding with LOAD status. We found a significant association between the interaction term of inbreeding and $A P O E$ with LOAD status $\left(P=4.04 \times 10^{3}\right)$.

A higher inbreeding coefficient was associated with an increased risk of LOAD, which is consistent with findings in other complex diseases, including coronary heart disease, stroke, cancer, depression, asthma, type 2 diabetes, and gout. ${ }^{32}$ Contrary to the study of other complex diseases, inbreeding does not significantly affect the age at onset of LOAD, but a tendency for lower age at onset was observed with increased inbreeding in the data set (Tables 3 and 4).

\section{DISCUSSION}

Our findings regarding the extent of inbreeding in Caribbean Hispanics were consistent with previous reports in this population. ${ }^{19}$ Accounting for admixture, we show that the true extent of inbreeding is less than second-cousin mating but greater than that of outbred populations in which consanguineous marriages occur at a low frequency. The range of inbreeding rates in Canada (Roman Catholics) is 0.00004-0.00007, in the United States (Roman Catholics) it is $0-0.0008$, in Latin America it is $0-0.003$, in southern Europe it is 0.001-0.002, and in Japan it is approximately $0.005 .{ }^{33}$ As compared with that of the Samaritans $(F=0.04)$, a 3,000-year-old genetic-isolate population comprising only 500 people, the observed inbreeding in Caribbean Hispanics is at an intermediate level. ${ }^{34}$ Despite the higher risk that inbreeding might confer in complex lateonset traits, ${ }^{7,8}$ it has not been well-studied in LOAD. In this study we demonstrate a statistically significant association of the extent of inbreeding with $\mathrm{AD}$ risk. This is consistent with the hypothesis that a significant proportion of risk in complex diseases such as LOAD could be mediated through multiple causal recessive loci resulting from increased homozygosity in inbred subjects. This is also consistent with our previous finding of larger $\mathrm{ROH}$ and higher numbers of $\mathrm{ROH}$ in LOAD patients $(n=559)$ versus controls $(n=554)^{19}$ from the same population. In the Caribbean Hispanic population, the authors detected an association between LOAD and a larger genome-wide mean ROH size $(P=0.0039)$, which was stronger with familial LOAD $(P=0.0005)$; however, studies of Caucasian data sets have not reported an increased burden of $\mathrm{ROH}$ in $\mathrm{AD}^{20,35} \mathrm{~A}$ likely explanation is that the significant inbreeding in the Caribbean Hispanics as detailed in this report increases the likelihood of recessive alleles in affected subjects resulting in longer $\mathrm{ROH}$ and higher numbers of $\mathrm{ROH}$ in the genome. Interestingly, the authors also noted that total $\mathrm{ROH}$ size was twice as long in the European Hispanic subset versus the African Hispanic subset, which is corroborated by our observation of a higher inbreeding coefficient in the Caucasian subset of the data (Table 2). The higher level of inbreeding in the Caucasians is likely to render larger regions in the genome homozygous as compared with those of the African subset of this population.

The high degree of inbreeding and presence of long $\mathrm{ROH}$ combined with higher frequency of $\mathrm{AD}$ in the Caribbean Hispanic population compared with Caucasians ${ }^{22}$ suggests that there may be one or more recessive loci mediating $\mathrm{AD}$ risk in this population. Low-frequency mutations are hypothesized to confer greater risk for disease than common variants by collectively accounting for substantial fractions of common disease heritability. ${ }^{36}$ Inbred populations with few founders, such as the Caribbean Hispanics, share large chromosomal segments recurring among relatives, and otherwise rare alleles can be observed repeatedly in multiple individuals. This reduces falsepositive findings due to sequencing errors that can be difficult to identify in isolated cases from outbred populations.

\section{SUPPLEMENTARY MATERIAL}

Supplementary material is linked to the online version of the paper at http://www.nature.com/gim

\section{ACKNOWLEDGMENTS}

This work was supported by grants from the National Institute of Aging (R37AG015473 (EFIGA study) and P01AG007232, R01AG037212 (WHICAP study)), the Wellcome Trust, the Medical Research Council, the Canadian Institutes of Health Research, the Ontario Research Fund, and the Alzheimer Society of Ontario.

\section{DISCLOSURE}

The authors declare no conflict of interest.

\section{REFERENCES}

1. Bittles $A H$, Neel JV. The costs of human inbreeding and their implications for variations at the DNA level. Nat Genet 1994;8:117-121.

2. Charlesworth $B, C$ harlesworth $D$. The genetic basis of inbreeding depression. Genet Res 1999;74:329-340.

3. Bittles AH, Savithri HS, Venkatesha Murthy H, et al. Consanguineous marriage, a familiar story full of surprises. In: Macbeth $\mathrm{H}$ and Shetty $\mathrm{P}$ (eds.). Health and Ethnicity. Taylor \& Francis: London, 2001:68-78.

4. Wright AF, Hastie ND. Complex genetic diseases: controversy over the Croesus code. Genome Biol 2001;2:COMMENT2007.

5. Bittles AH, Mason WM, Greene J, Rao NA. Reproductive behavior and health in consanguineous marriages. Science 1991;252:789-794. 
6. Bittles AH, Black ML. The impact of consanguinity on neonatal and infant health. Early Hum Dev 2010;86:737-741.

7. Charlesworth B, Hughes KA. Age-specific inbreeding depression and components of genetic variance in relation to the evolution of senescence. Proc Natl Acad Sci USA 1996;93:6140-6145.

8. Hughes KA, Alipaz JA, Drnevich JM, Reynolds RM. A test of evolutionary theories of aging. Proc Natl Acad Sci USA 2002;99:14286-14291.

9. Assié G, LaFramboise T, Platzer P, Eng C. Frequency of germline genomic homozygosity associated with cancer cases. JAMA 2008;299: 1437-1445.

10. Bacolod MD, Schemmann GS, Wang S, et al. The signatures of autozygosity among patients with colorectal cancer. Cancer Res 2008;68:2610-2621.

11. Enciso-Mora V, Hosking FJ, Houlston RS. Risk of breast and prostate cancer is not associated with increased homozygosity in outbred populations. Eur J Hum Genet 2010;18:909-914.

12. Hosking FJ, Papaemmanuil E, Sheridan E, et al. Genome-wide homozygosity signatures and childhood acute lymphoblastic leukemia risk. Blood 2010;115:4472-4477.

13. Siraj $A K$, Khalak HG, Sultana $M$, et al. Colorectal cancer risk is not associated with increased levels of homozygosity in Saudi Arabia. Genet Med 2012;14:720-728.

14. Spain SL, Cazier JB, Houlston R, Carvajal-Carmona L, Tomlinson I; CORGI Consortium. Colorectal cancer risk is not associated with increased levels of homozygosity in a population from the United Kingdom. Cancer Res 2009;69:7422-7429.

15. Wingo TS, Lah JJ, Levey Al, Cutler DJ. Autosomal recessive causes likely in earlyonset Alzheimer disease. Arch Neurol 2012;69:59-64.

16. Farrer LA, Bowirrat A, Friedland RP, Waraska K, Korczyn AD, Baldwin CT. Identification of multiple loci for Alzheimer disease in a consanguineous IsraeliArab community. Hum Mol Genet 2003;12:415-422.

17. Sherva R, Baldwin CT, Inzelberg R, et al. Identification of novel candidate genes for Alzheimer's disease by autozygosity mapping using genome wide SNP data. J Alzheimers Dis 2011;23:349-359.

18. Vézina H, Heyer E, Fortier I, Ouellette G, Robitaille Y, Gauvreau D. A genealogical study of Alzheimer disease in the Saguenay region of Quebec. Genet Epidemiol 1999;16:412-425.

19. Ghani M, Sato C, Lee JH, et al. Evidence of recessive Alzheimer disease loci in a Caribbean Hispanic data set: genome-wide survey of runs of homozygosity. JAMA Neurol 2013;70:1261-1267.

20. Curtis $D$, Vine AE, Knight J. Study of regions of extended homozygosity provides a powerful method to explore haplotype structure of human populations. Ann Hum Genet 2008;72(Pt 2):261-278.

21. Nalls MA, Guerreiro RJ, Simon-Sanchez J, et al. Extended tracts of homozygosity identify novel candidate genes associated with late-onset Alzheimer's disease. Neurogenetics 2009;10:183-190.
22. Vardarajan BN, Faber KM, Bird TD, et al. Age-specific incidence rates for dementia and Alzheimer disease in NIA-LOAD/NCRAD and EFIGA families: National Institute on Aging Genetics Initiative for Late-Onset Alzheimer Disease/National Cell Repository for Alzheimer Disease (NIA-LOAD/NCRAD) and Estudio Familiar de Influencia Genetica en Alzheimer (EFIGA). JAMA Neurol 2014;71:315-323.

23. Tang MX, Stern Y, Marder K, et al. The APOE-epsilon4 allele and the risk of Alzheimer disease among African Americans, whites, and Hispanics. JAMA 1998;279:751-755.

24. Romas SN, Santana V, Williamson J, et al. Familial Alzheimer disease among Caribbean Hispanics: a reexamination of its association with APOE. Arch Neurol 2002;59:87-91.

25. McKhann G, Drachman D, Folstein M, Katzman R, Price D, Stadlan EM. Clinical diagnosis of Alzheimer's disease: report of the NINCDS-ADRDA Work Group under the auspices of Department of Health and Human Services Task Force on Alzheimer's Disease. Neurology 1984;34:939-944.

26. Lee JH, Cheng R, Barral S, et al. Identification of novel loci for Alzheimer disease and replication of CLU, PICALM, and BIN1 in Caribbean Hispanic individuals. Arch Neurol 2011;68:320-328.

27. Laurie CC, Doheny KF, Mirel DB, et al.; GENEVA Investigators. Quality control and quality assurance in genotypic data for genome-wide association studies. Genet Epidemiol 2010;34:591-602.

28. Purcell S, Neale B, Todd-Brown K, et al. PLINK: a tool set for whole-genome association and population-based linkage analyses. Am J Hum Genet 2007;81:559-575.

29. Yang J, Lee SH, Goddard ME, Visscher PM. GCTA: a tool for genome-wide complex trait analysis. Am J Hum Genet 2011;88:76-82.

30. Thornton T, Tang H, Hoffmann TJ, Ochs-Balcom HM, Caan BJ, Risch N. Estimating kinship in admixed populations. Am J Hum Genet 2012;91: 122-138.

31. Alexander DH, Novembre J, Lange K. Fast model-based estimation of ancestry in unrelated individuals. Genome Res 2009;19:1655-1664.

32. Rudan I, Rudan D, Campbell H, et al. Inbreeding and risk of late onset complex disease. J Med Genet 2003;40:925-932.

33. Bittles AH. Consanguineous Marriage: Current Global Incidence and Its Relevance to Demographic Research. University of Michigan: Ann Arbor, MI, 1990.

34. Thompson MW, McInnes RR, Willard HF. Thompson and Thompson: Genetics in Medicine. WB Saunders: Toronto, Canada, 1991.

35. Sims R, Dwyer $S$, Harold $D$, et al. No evidence that extended tracts of homozygosity are associated with Alzheimer's disease. Am J Med Genet B Neuropsychiatr Genet 2011;156B:764-771.

36. Sebat J, Levy DL, McCarthy SE. Rare structural variants in schizophrenia: one disorder, multiple mutations; one mutation, multiple disorders. Trends Genet 2009;25:528-535. 